\title{
Automorphisms of quantum and classical Poisson algebras
}

\author{
J. Grabowski and N. Poncin
}

\begin{abstract}
We prove Pursell-Shanks type results for the Lie algebra $\mathcal{D}(M)$ of all linear differential operators of a smooth manifold $M$, for its Lie subalgebra $\mathcal{D}^{1}(M)$ of all linear first-order differential operators of $M$ and for the Poisson algebra $S(M)=\operatorname{Pol}\left(T^{*} M\right)$ of all polynomial functions on $T^{*} M$, the symbols of the operators in $\mathcal{D}(M)$. Chiefly, however, we provide explicit formulas completely describing the automorphisms of the Lie algebras $\mathcal{D}^{1}(M), S(M)$ and $\mathcal{D}(M)$.
\end{abstract}

\section{Introduction}

The classical result of Pursell and Shanks [PS54], which states that the Lie algebra of smooth vector fields of a smooth manifold characterizes the smooth structure of the variety, is the starting point of a multitude of papers.

There are similar results in particular geometric situations - for instance for Hamiltonian, contact or group invariant vector fields - for which specific tools have been constructed [Omo76, Abe82, AG90, HM93], in the case of Lie algebras of vector fields that are modules over the corresponding rings of functions [Ame75, Gra78, Skr87], as well as for the Lie algebra of (not leaf but) foliation preserving vector fields [Gra93].

The initial objective of the present paper was to prove that the Lie algebra $\mathcal{D}(M)$ of all linear differential operators $D: C^{\infty}(M) \rightarrow C^{\infty}(M)$ of a smooth manifold $M$, determines the smooth structure of $M$. Beyond this conclusion, we present a description of all automorphisms of the Lie algebra $\mathcal{D}(M)$ and even of the Lie subalgebra $\mathcal{D}^{1}(M)$ of all linear first-order differential operators of $M$ and of the Poisson algebra $S(M)=\operatorname{Pol}\left(T^{*} M\right)$ of polynomial functions on the cotangent bundle $T^{*} M$ (the symbols of the operators in $\mathcal{D}(M)$ ), the automorphisms of the two last algebras of course being canonically related with those of $\mathcal{D}(M)$. In each situation we obtain an explicit formula, for instance - in the case of $\mathcal{D}(M)$ - in terms of the automorphism of $\mathcal{D}(M)$ implemented by a diffeomorphism of $M$, the conjugation-automorphism of $\mathcal{D}(M)$ and the automorphism of $\mathcal{D}(M)$ generated by the derivation of $\mathcal{D}(M)$ associated to a closed 1-form of $M$.

In the first part of our work, the approach is purely algebraic. In $\S 2$, we heave $\mathcal{D}(M)$ and $S(M)$ on a general algebraic level and define the notions 'quantum Poisson algebra' $\mathcal{D}$ and 'classical Poisson algebra' $S$, the classical limit of $\mathcal{D}$. In $\S 3$ we show that if two (quantum or classical) Poisson algebras are isomorphic as Lie algebras, their 'basic algebras of functions' are isomorphic as associative algebras - an algebraic Shanks-Pursell type result, which naturally implies our previously described initial goal. The leading idea of the proof is the algebraic characterization, under a minimal

Received 18 November 2002, accepted in final form 20 February 2003.

2000 Mathematics Subject Classification 17B63 (primary), 13N10, 16S32, 17B40, 17B65, 53D17 (secondary).

Keywords: algebraic characterization, smooth manifold, automorphisms, differential operators, smooth functions, symbols.

This work was partially supported by NATO Grant RD/DOC/020204-3 and by MCESR Grant RD/C.U.L./02-010. This journal is (C) Foundation Compositio Mathematica 2004. 


\section{J. Grabowski and N. Poncin}

condition, of functions as those $D \in \mathcal{D}\left(\right.$ or $P \in S$ ) for which $\operatorname{ad}_{D}\left(\operatorname{respectively~}_{P}\right)$ is locally nilpotent.

In the second part of the paper, we switch to the concrete geometric context. In this introduction, we have confined ourselves to a very rough description of the quite technical computations of $\S 7$, which give all the automorphisms of $\mathcal{D}(M)$, calculations based on the result in the $S(M)$-case $(\S 6)$, itself founded on the $\mathcal{D}^{1}(M)$-case $(\S 5)$. The utilization - in addition to the algebra hierarchy just mentioned - of the preliminarily detected conjugation- and derivation-automorphisms $(\S 4)$ and the suitable use of the normal ordering method (i.e. the local polynomial representation of differential operators), allow the problem to be reduced to the determination of intertwining operators between some modules of the Lie algebra of vector fields and to be concluded.

\section{Definitions and tools}

By a quantum Poisson algebra we mean an associative filtered algebra $\mathcal{D}=\bigcup_{i=0}^{\infty} \mathcal{D}^{i}, \mathcal{D}^{i} \subset \mathcal{D}^{i+1}$, $\mathcal{D}^{i} \cdot \mathcal{D}^{j} \subset \mathcal{D}^{i+j}$ (where $\cdot$ denotes the multiplication of $\mathcal{D}$ ), with unit 1 over a field $K$ of characteristic 0 , such that $\left[\mathcal{D}^{i}, \mathcal{D}^{j}\right] \subset \mathcal{D}^{i+j-1}$, where $[\cdot, \cdot]$ is the commutator bracket and where $\mathcal{D}^{i}=\{0\}$ for $i<0$, by convention.

It is obvious that $\mathcal{A}=\mathcal{D}^{0}$ is a commutative subalgebra of $\mathcal{D}$ (we call it the basic algebra of $\mathcal{D}$ ) and $\mathcal{D}^{1}$ a Lie subalgebra of $\mathcal{D}$. We refer to elements $k$ of $K$, naturally embedded in $\mathcal{A}$ or $\mathcal{D}$ by $k \in K \rightarrow k 1 \in \mathcal{A} \subset \mathcal{D}$, as constants, to elements $f$ of $\mathcal{A}$ as functions and to elements $D$ of $\mathcal{D}$ as differential operators. It is easily seen that every element $D \in \mathcal{D}^{1}$, i.e. every first-order differential operator, induces a derivation $\hat{D} \in \operatorname{Der}(\mathcal{A})$ of $\mathcal{A}$ by $\hat{D}(f)=[D, f]$.

By a classical Poisson algebra we mean a commutative associative algebra with an $\mathbb{N}$-gradation $S=\bigoplus_{i=0}^{\infty} S_{i}, S_{i} S_{j} \subset S_{i+j}$, with unit 1 over a field $K$ of characteristic 0 , equipped with a Poisson bracket $\{\cdot, \cdot\}$ such that $\left\{S_{i}, S_{j}\right\} \subset S_{i+j-1}$. Of course, we can think of $S$ as of a $\mathbb{Z}$-graded algebra putting $S_{i}=\{0\}$ for $i<0$ and as a filtered algebra putting $S^{i}=\bigoplus_{k \leqslant i} S_{k}$. As in the case of the quantum Poisson algebra, $\mathcal{A}=S_{0}$ is an associative and Lie-commutative subalgebra of $S$ (the basic algebra) and $S^{1}$ is a Lie subalgebra of $(S,\{\cdot, \cdot\})$ acting on $\mathcal{A}$ by derivations.

An operator $\phi \in \operatorname{Hom}_{K}\left(V_{1}, V_{2}\right)$ between $\mathbb{N}$-filtered vector spaces respects the filtration if $\phi\left(V_{1}^{i}\right) \subset$ $V_{2}^{i}$ and is lowering if $\phi\left(V_{1}^{i}\right) \subset V_{2}^{i-1}$.

Quantum Poisson algebras induce canonically classical Poisson algebras as follows. For a quantum Poisson algebra $\mathcal{D}$, consider the graded vector space $S(\mathcal{D})=\bigoplus_{i \in \mathbb{Z}} S_{i}(\mathcal{D}), S_{i}(\mathcal{D})=\mathcal{D}^{i} / \mathcal{D}^{i-1}$. We have the obvious canonical surjective map $\sigma: \mathcal{D} \rightarrow S$, the principal-symbol map. Note that $\sigma(\mathcal{A})=\mathcal{A}=S_{0}$. We denote the projection of $\sigma(D)$ to $S_{j}$ by $\sigma(D)_{j}$.

Since for each non-zero differential operator $D \in \mathcal{D}$, there is a single $i=\operatorname{deg}(D) \in \mathbb{Z}$ such that $D \in \mathcal{D}^{i} \backslash \mathcal{D}^{i-1}, \sigma(D)_{j}=0$ if $j \neq \operatorname{deg}(D)$ and $\sigma(D)_{\operatorname{deg}(D)}=\sigma(D)$. We set for $\dot{D}_{1} \in S_{i}$ with $\dot{D}_{1}=\sigma\left(D_{1}\right)$ and $\dot{D}_{2} \in S_{j}$ with $\dot{D}_{2}=\sigma\left(D_{2}\right)$,

$$
\dot{D}_{1} \dot{D}_{2}=\sigma\left(D_{1} \cdot D_{2}\right)_{i+j}, \quad\left\{\dot{D}_{1}, \dot{D}_{2}\right\}=\sigma\left(\left[D_{1}, D_{2}\right]\right)_{i+j-1} .
$$

It is easy to see that these definitions do not depend on the choice of the representatives $D_{1}$ and $D_{2}$ and that we get a classical Poisson algebra with the same basic algebra $\mathcal{A}$. We call this classical Poisson algebra the classical limit of the quantum Poisson algebra $\mathcal{D}$. We can formulate this as follows.

Theorem 1. For every quantum Poisson algebra $\mathcal{D}$, there is a unique classical Poisson algebra structure on the graded vector space $S(\mathcal{D})$ such that

$$
\sigma\left(D_{1}\right) \sigma\left(D_{2}\right)=\sigma\left(D_{1} \cdot D_{2}\right) \operatorname{deg}\left(D_{1}\right)+\operatorname{deg}\left(D_{2}\right)
$$


and

$$
\left\{\sigma\left(D_{1}\right), \sigma\left(D_{2}\right)\right\}=\sigma\left(\left[D_{1}, D_{2}\right]\right) \operatorname{deg}\left(D_{1}\right)+\operatorname{deg}\left(D_{2}\right)-1
$$

for each $D_{1}, D_{2} \in \mathcal{D}$. In particular,

$$
\left\{\sigma\left(D_{1}\right), \sigma\left(D_{2}\right)\right\}=\sigma\left(\left[D_{1}, D_{2}\right]\right) \quad \text { or } \quad\left\{\sigma\left(D_{1}\right), \sigma\left(D_{2}\right)\right\}=0 .
$$

Corollary 1. For $D_{1}, D_{2}, \ldots, D_{n} \in \mathcal{D}$, if

$$
\left[D_{1},\left[D_{2}, \ldots,\left[D_{n-1}, D_{n}\right]\right]\right]=0
$$

then

$$
\left\{\sigma\left(D_{1}\right),\left\{\sigma\left(D_{2}\right), \ldots,\left\{\sigma\left(D_{n-1}\right), \sigma\left(D_{n}\right)\right\}\right\}\right\}=0
$$

Note that every linear map $\Phi: \mathcal{D}_{1} \rightarrow \mathcal{D}_{2}$ between two quantum Poisson algebras, which respects the filtration, canonically induces a linear map $\tilde{\Phi}: S\left(\mathcal{D}_{1}\right) \rightarrow S\left(\mathcal{D}_{2}\right)$, which respects the gradation, by $\tilde{\Phi}(\sigma(D))=\sigma(\Phi(D))$. In view of Theorem 1, it is easy to see that if such $\Phi$ is a homomorphism of associative (respectively Lie) structure, then $\tilde{\Phi}$ is a homomorphism of associative (respectively Lie) structure.

A classical Poisson algebra $S$ is said to be non-singular if $\left\{S^{1}, \mathcal{A}\right\}=\mathcal{A}$. The Poisson algebra $S$ is called symplectic if constants are the only central elements of $(S,\{\cdot, \cdot\})$ and distinguishing if for any $P \in S$ we have that

$$
\forall f \in \mathcal{A}, \exists n \in \mathbb{N}: \underbrace{\{P,\{P, \ldots,\{P}_{n}, f\}\}\}=0 \Rightarrow P \in \mathcal{A} .
$$

A quantum Poisson algebra is called non-singular (respectively symplectic or distinguishing), if its classical limit is a non-singular (respectively symplectic or distinguishing) classical Poisson algebra.

Proposition 1. For any quantum Poisson algebra $\mathcal{D}$ :

a) $\mathcal{D}$ is non-singular if and only if $\left[\mathcal{D}^{1}, \mathcal{A}\right]=\mathcal{A}$;

b) if $\mathcal{D}$ is symplectic, then the constants are the only central elements in $\mathcal{D}$;

c) if $\mathcal{D}$ is distinguishing, then for any $D \in \mathcal{D}$ we have that

$$
\forall f \in \mathcal{A}, \exists n \in \mathbb{N}: \underbrace{[D,[D, \ldots,[D}_{n}, f]]]=0 \Rightarrow D \in \mathcal{A} .
$$

Proof. It is obvious that

$$
\left[\mathcal{D}^{1}, \mathcal{A}\right]=\sigma\left(\left[\mathcal{D}^{1}, \mathcal{A}\right]\right)=\left\{S^{1}(\mathcal{D}), \mathcal{A}\right\}
$$

which proves item a. To prove part b, it suffices to observe that the center of the Lie algebra $S(\mathcal{D})$ contains the image of the center of $\mathcal{D}$ by the map $\sigma$. Finally, in view of Corollary 1 ,

$$
\underbrace{[D,[D, \ldots,[D}_{n}, f]]]=0
$$

implies

$$
\underbrace{\{\sigma(D),\{\sigma(D), \ldots,\{\sigma(D)}_{n}, f\}\}\}=0
$$

and part c follows.

Example 1. A standard example of a quantum Poisson algebra is the algebra $\mathcal{D}(M)$ of differential operators $D: C^{\infty}(M) \rightarrow C^{\infty}(M)$ associated with a manifold $M$. Its classical limit $S(M)$ is the Poisson algebra $\operatorname{Pol}\left(T^{*} M\right)$ of polynomials on the cotangent bundle $T^{*} M$ (i.e. of the smooth functions on $T^{*} M$ that are polynomial along the fibers) with the standard symplectic Poisson bracket on $T^{*} M$. 


\section{J. Grabowski and N. Poncin}

We can also view $S(M)$ as the algebra of symmetric contravariant tensors on $M$ with the symmetric Schouten bracket. We have a canonical splitting $\mathcal{D}(M)=\mathcal{A} \oplus \mathcal{D}_{c}(M)$, where $\mathcal{A}=C^{\infty}(M)$ and where $\mathcal{D}_{c}(M)$ is the algebra of differential operators vanishing on constants $\left(D \in \mathcal{D}_{c}(M)\right.$ if and only if $D(1)=0)$. If $\mathcal{D}_{c}^{i}(M)=\mathcal{D}^{i}(M) \cap \mathcal{D}_{c}(M)(i \geqslant 0)$, we also have $\mathcal{D}^{i}(M)=\mathcal{A} \oplus \mathcal{D}_{c}^{i}(M)$. It is clear that $\mathcal{D}_{c}^{0}(M)=0$ and that $\mathcal{D}_{c}^{1}(M)$ is the Lie algebra $\operatorname{Der}(\mathcal{A})$ of derivations of $\mathcal{A}$, i.e. the Lie algebra $\operatorname{Vect}(M)$ of vector fields on $M$. Note that the Lie algebras $\mathcal{D}^{1}(M)$ and $S^{1}(M)$ are both isomorphic to $\operatorname{Vect}(M) \oplus C^{\infty}(M)$ with the bracket $[X+f, Y+g]=[X, Y]+(X(g)-Y(f))$.

The quantum Poisson algebra $\mathcal{D}(M)$ is easily seen to be non-singular and symplectic. In the next section we show that it is distinguishing.

Example 2. The above example can be extended to the case of the quantum Poisson algebra of differential operators on a given associative commutative algebra $\mathcal{A}$ with unit 1 . The corresponding differential calculus has been developed and extensively studied by Vinogradov [Vin72].

To investigate the algebra $\mathcal{D}(M)$ of differential operators we need some preparation. Let us look at local representations of differential operators and the formal calculus (see, e.g., [DWL83, Pon01]).

Consider an open subset $U$ of $\mathbb{R}^{n}$, two real finite-dimensional vector spaces $E$ and $F$ and some local operator

$$
O \in \mathcal{L}\left(C^{\infty}(U, E), C^{\infty}(U, F)\right)_{\text {loc }} .
$$

The operator is fully defined by its values on the products $f e, f \in C^{\infty}(U), e \in E$. A well known theorem of Peetre (see [Pee59]) states that it has the form

$$
O(f e)=\sum_{\alpha} O_{\alpha}\left(\partial^{\alpha}(f e)\right)=\sum_{\alpha} O_{\alpha}(e) \partial^{\alpha} f
$$

where $\partial_{x}^{\alpha}=\partial_{x^{1}}^{\alpha^{1}} \cdots \partial_{x^{m}}^{\alpha^{m}}$ and $O_{\alpha} \in C^{\infty}(U, \mathcal{L}(E, F))$. Moreover, the coefficients $O_{\alpha}$ are well determined by $O$ and the series is locally finite (it is finite if $U$ is relatively compact).

We symbolize the partial derivative $\partial^{\alpha} f$ by the monomial $\xi^{\alpha}=\xi_{1}^{\alpha^{1}} \cdots \xi_{m}^{\alpha^{m}}$ in the components $\xi_{1}, \ldots, \xi_{m}$ of some linear form $\xi \in\left(\mathbb{R}^{n}\right)^{*}$, or - at least mentally - even by $\xi^{\alpha} f$, if this is necessary to avoid confusion. The operator $O$ is thus represented by the polynomial

$$
\mathcal{O}(\xi ; e)=\sum_{\alpha} O_{\alpha}(e) \xi^{\alpha}
$$

When identifying the space $\operatorname{Pol}\left(\left(\mathbb{R}^{n}\right)^{*}\right)$ of polynomials on $\left(\mathbb{R}^{n}\right)^{*}$ with the space $\bigvee \mathbb{R}^{n}$ of symmetric contravariant tensors of $\mathbb{R}^{n}$, we have $\mathcal{O} \in C^{\infty}\left(U, \bigvee \mathbb{R}^{n} \otimes \mathcal{L}(E, F)\right)$. Let us emphasize that the form $\xi$ symbolizes the derivatives in $O$ that act on the argument $f e \in C^{\infty}(U, E)$, while $e \in E$ represents this argument. In the following, we no longer use different notation for the operator $O$ and its representative polynomial $\mathcal{O}$; in order to simplify notations, it is helpful to use the same typographical sign even when referring to the argument $f e$ and its representation $e$.

For instance, let us look for the local representation of the Lie derivative of a differential operator (it is well known that $L_{X} D=[X, D]\left(X \in \operatorname{Vect}(M), D \in \mathcal{D}^{i}(M)\right.$ or $\left.D \in \mathcal{D}_{c}^{i}(M)\right)$ defines a module structure over $\operatorname{Vect}(M)$ on $\mathcal{D}^{i}(M)$ and $\mathcal{D}_{c}^{i}(M)$, respectively). If $D \in \mathcal{D}(M)$, its restriction $\left.D\right|_{U}$ (or simply $D$, if no confusion is possible) to a domain $U$ of local coordinates of $M$ is a local operator from $C^{\infty}(U)$ into $C^{\infty}(U)$ that is represented by $D(f) \simeq D(\xi ; 1)=D(\xi)$, where $f \in C^{\infty}(U)$ and where $\xi$ represents the derivatives acting on $f$. The Lie derivative of $D(f)$ with respect to a vector field $X \in C^{\infty}\left(U, \mathbb{R}^{n}\right)$, is then represented by $L_{X}(D(f)) \simeq\langle X, \eta+\xi\rangle D(\xi)$. Here, $\eta \in\left(\mathbb{R}^{n}\right)^{*}$ is associated to $D$ and $\langle X, \eta+\xi\rangle$ denotes the evaluation of $X \in \mathbb{R}^{n}$ on $\eta+\xi$. When associating $\zeta$ to $X$, we get $D\left(L_{X} f\right) \simeq\langle X, \xi\rangle D(\xi+\zeta)$ and

$$
\left(L_{X} D\right)(f) \simeq\langle X, \eta\rangle D(\xi)-\langle X, \xi\rangle \tau_{\zeta} D(\xi),
$$

where $\tau_{\zeta} D(\xi)=D(\xi+\zeta)-D(\xi)$. 


\section{Automorphisms of QuAntum and Classical Poisson algebras}

\section{Algebraic characterization of a manifold}

Theorem 2. The quantum Poisson algebra $\mathcal{D}(M)$ of differential operators on $C^{\infty}(M)$ is distinguishing (i.e. the classical Poisson algebra $S(M)$ is distinguishing).

Proof. Since for $P, Q \in S=\operatorname{Pol}\left(T^{*} M\right),\{P, Q\}=H_{P} . Q$, where $H_{P}$ is the Hamiltonian vector field of $P$, we have to prove that if $P \in S \backslash \mathcal{A}\left(\mathcal{A}=C^{\infty}(M)\right)$, there is a function $f \in \mathcal{A}$ such that for every integer $n \in \mathbb{N},\left(H_{P}\right)^{n} \cdot f \neq 0$.

If $\left(U,\left(x^{1}, \ldots, x^{n}\right)\right)$ is a chart of $M$, then $H_{P}$ has in the associated Darboux chart $\left(T^{*} U,\left(x^{1}, \ldots, x^{n}, \xi_{1}, \ldots, \xi_{n}\right)\right)$ the classical expression $H_{P}=\bar{\partial}_{i} P \partial_{i}-\partial_{i} P \bar{\partial}_{i}$, where $\bar{\partial}_{i}=\partial / \partial \xi_{i}$ and $\partial_{i}=\partial / \partial x^{i}$. It follows from the hypothesis $P \in S \backslash \mathcal{A}$ that $\bar{\partial}_{i} P \partial_{i} \neq 0$ for at least one chart $U$ of $M$. In order to simplify notation, we write in the associated Darboux chart $T^{*} U, H_{P}=F^{i} \partial_{i}+G_{i} \bar{\partial}_{i}$, with $F^{i} \partial_{i} \neq 0$.

First note that, for an arbitrary neighborhood $] a, b\left[\right.$ of an arbitrary point $x_{0} \in \mathbb{R}$, it is possible to construct a sequence $\left.x_{1}, x_{2}, \ldots \in\right] a, b\left[\right.$ with limit $x_{0}$ and a function $h \in C^{\infty}(\mathbb{R})$ such that, if $d_{x}^{k} h$ denotes the $k$ th derivative of $h$,

$$
\left(d_{x}^{k} h\right)\left(x_{n}\right) \begin{cases}=0, & \text { for all } k \in\{0, \ldots, n-1\} \\ \neq 0, & \text { for } k=n .\end{cases}
$$

Indeed, set $d=\left(b-x_{0}\right) / 2, x_{n}=x_{0}+d / n\left(n \in \mathbb{N}^{*}\right), \delta_{n}=x_{n}-x_{n+1}$, and $\left.V_{n}=\right] x_{n}-\delta_{n} / 2, x_{n}+\delta_{n} / 2[$. It is clear that the intersections $V_{n} \cap V_{n+1}$ are empty. Now take smooth functions $\alpha_{n}$ with value 1 around $x_{n}$ and compact support in $V_{n}$ and define smooth functions $h_{n}$ by $h_{n}(x)=\left(x-x_{n}\right)^{n} \alpha_{n}(x)$. We easily see that $\left(d_{x}^{k} h_{n}\right)\left(x_{n}\right)$ vanishes for all $k \in\{0, \ldots, n-1\}$ and does not for $k=n$. Finally, the function $h$ defined by $h(x)=\sum_{n=1}^{\infty} h_{n}(x)$ has all the desired properties.

When returning to the initial problem, remark that at least one $F^{i}$ does not vanish, say $F^{1}$. If its value at some point $\left(x_{0}, \xi_{0}\right) \in T^{*} U$ is non-zero, the function $F^{1}\left(\cdot, \xi_{0}\right) \in C^{\infty}(U)$ is non-zero on some neighborhood $V$ of $x_{0}$.

In the following, the coordinates $\left(x^{1}, \ldots, x^{n}\right)$ of a point $x \in U$ will be denoted by $\left(x^{1}, x^{\prime \prime}\right) \in$ $\mathbb{R} \times \mathbb{R}^{n-1}$. Consider now $V$ as an open subset of $\mathbb{R}^{n}$, introduce the section $V^{1}=\left\{x^{1}:\left(x^{1}, x_{0}^{\prime \prime}\right) \in V\right\}$ of $V$ at the level $x_{0}^{\prime \prime}$ and construct the previously described sequence $x_{n}^{1}$ and function $h$ in this neighborhood $V^{1}$ of $x_{0}^{1}$. The sequence defines a sequence $x_{n}$ in $V$ with the limit $x_{0}$ and the function defines a function still denoted by $h$ in $C^{\infty}(V)$.

When multiplying this $h$ by a smooth $\alpha$, which has the value 1 in a neighborhood of the points $x_{n}$ and is compactly supported in $V$, we get the function $f \in C^{\infty}(M)$ that we have to construct. Indeed, for every $n$,

$$
\left(\left(H_{P}\right)^{n} \cdot f\right)\left(x_{n}, \xi_{0}\right)=\left(\left(F^{i} \partial_{i}+G_{i} \bar{\partial}_{i}\right)^{n} h\right)\left(x_{n}, \xi_{0}\right) .
$$

The function on the right-hand side is a sum of terms in the $\partial_{i} h, \partial_{i_{1}} \partial_{i_{2}} h, \ldots, \partial_{i_{1}} \cdots \partial_{i_{n}} h$ and the maximal order terms are $F^{i_{1}} \cdots F^{i_{n}} \partial_{i_{1}} \cdots \partial_{i_{n}} h$. All the terms of order less than $n$ vanish, since the derivatives with respect to $x^{i}(i \neq 1)$ vanish and for $k<n,\left(d_{x^{1}}^{k} h\right)\left(x_{n}^{1}\right)=0$. The terms of maximal order $n$ also vanish, except $\left(F^{1}\right)^{n} d_{x^{1}}^{n} h$ that is non-zero at $\left(x_{n}, \xi_{0}\right)$.

For any Lie algebra $(\mathcal{L},[\cdot, \cdot])$, we denote the set of those $D \in \mathcal{L}$ for which $\operatorname{ad}_{D}$ is locally nilpotent by $\operatorname{Nil}(\mathcal{L})$ :

$$
\left.\left.\left.\operatorname{Nil}(\mathcal{L})=\{D \in \mathcal{L}: \forall D^{\prime} \in \mathcal{L}, \exists n \in \mathbb{N}: \underbrace{[D,[D, \ldots,[D}_{n}, D^{\prime}]\right]\right]=0\right\} .
$$

Proposition 2. If a quantum or classical Poisson algebra $\mathcal{L}$ with the basic algebra $\mathcal{A}$ is distinguishing, then:

a) $\operatorname{Nil}(\mathcal{L})=\mathcal{A}$; 


\section{J. Grabowski and N. Poncin}

b)

$$
\left\{P \in S:\{P, \mathcal{A}\} \subset S_{i}\right\}=S_{i+1} \oplus \mathcal{A} \quad(i \geqslant-1)
$$

in the case $\mathcal{L}=S$ is classical; in particular,

$$
\begin{aligned}
& \left\{P \in S:\{P, \mathcal{A}\} \subset S^{i}\right\}=S^{i+1} \quad(i \geqslant-1) ; \\
& \text { c) } \quad\left\{D \in \mathcal{D}:[D, \mathcal{A}] \subset \mathcal{D}^{i}\right\}=\mathcal{D}^{i+1} \quad(i \geqslant-1)
\end{aligned}
$$

in the case $\mathcal{L}=\mathcal{D}$ is quantum.

Proof. a) This is obvious for classical and, in view of Proposition 1, also for quantum Poisson algebras.

b) Since $\{P, \mathcal{A}\} \subset S \ominus S_{i}$, for any $P \in S \ominus S_{i+1}$, the inclusion $\{P, \mathcal{A}\} \subset S_{i}$ for such $P$ implies $\{P, \mathcal{A}\}=0$, so $P \in \mathcal{A}$.

c) If $D \in \mathcal{D} \backslash \mathcal{D}^{i+1}$ and $[D, \mathcal{A}] \subset \mathcal{D}^{i}(i \geqslant-1)$, then $\{\sigma(D), \mathcal{A}\}=0$ and $\sigma(D) \in \mathcal{A}$, which is contradictory.

Now we start the studies on the properties of isomorphisms of quantum and classical Poisson algebras. We concentrate on the quantum level, since on the classical level all the considerations are analogous and even simpler.

Corollary 2. Every isomorphism $\Phi: \mathcal{D}_{1} \rightarrow \mathcal{D}_{2}$ of the Lie algebras $\left(\mathcal{D}_{i},[\cdot, \cdot]\right)$ for distinguishing quantum Poisson algebras $\mathcal{D}_{i}, i=1,2$, respects the filtration and induces an isomorphism $\tilde{\Phi}$ : $S\left(\mathcal{D}_{1}\right) \rightarrow S\left(\mathcal{D}_{2}\right), \tilde{\Phi}(\sigma(D))=\sigma(\Phi(D))$, of the corresponding classical limit Lie algebras.

Proof. It is obvious that $\Phi\left(\operatorname{Nil}\left(\mathcal{D}_{1}\right)\right)=\operatorname{Nil}\left(\mathcal{D}_{2}\right)$, i.e. $\Phi\left(\mathcal{A}_{1}\right)=\mathcal{A}_{2}$. Inductively, if $\Phi\left(\mathcal{D}_{1}^{i}\right) \subset \mathcal{D}_{2}^{i}$, then, for any $D \in \mathcal{D}_{1}^{i+1}$,

$$
\left[\Phi(D), \mathcal{A}_{2}\right]=\Phi\left(\left[D, \mathcal{A}_{1}\right]\right) \subset \mathcal{D}_{2}^{i}
$$

and $\Phi(D) \in \mathcal{D}_{2}^{i+1}$, by Proposition 2. Now, since $\Phi$ and $\Phi^{-1}$ respect the filtration, $\tilde{\Phi}$ is a linear isomorphism of $S_{1}$ onto $S_{2}$ which, as easily seen, is a Lie algebra isomorphism.

Denote by $C(\mathcal{D})$ the centralizer of $\operatorname{ad}_{\mathcal{A}}$ in $\operatorname{Hom}_{K}(\mathcal{D}, \mathcal{D})$ :

$$
\Psi \in C(\mathcal{D}) \Leftrightarrow\left[\Psi, \operatorname{ad}_{\mathcal{A}}\right]=0 .
$$

Note that multiplications $m_{f}: \mathcal{D} \ni D \rightarrow f \cdot D \in \mathcal{D}$ and $m_{f}^{\prime}: \mathcal{D} \ni D \rightarrow D \cdot f \in \mathcal{D}$ by elements $f \in \mathcal{A}$ belong to $C(\mathcal{D})$.

Theorem 3. Assume that $\mathcal{D}$ is a non-singular and distinguishing quantum Poisson algebra. Then any $\Psi \in C(\mathcal{D})$ respects the filtration and there is an $f \in \mathcal{A}$ and a lowering $\Psi_{1} \in C(\mathcal{D})$, such that

$$
\Psi=m_{f}+\Psi_{1} .
$$

Proof. $\left[\Psi, \operatorname{ad}_{\mathcal{A}}\right]=0$ means that

$$
[\Psi(D), f]=\Psi([D, f]),
$$

for all $D \in \mathcal{D}$ and all $f \in \mathcal{A}$. For $D \in \mathcal{A}$ we get $[\Psi(D), f]=0$, so $\Psi(D) \in \mathcal{A}$. Inductively, if $\Psi\left(\mathcal{D}^{i}\right) \subset \mathcal{D}^{i}$, then (4) implies $\left[\Psi\left(\mathcal{D}^{i+1}\right), f\right] \subset \mathcal{D}^{i}$ and $\Psi\left(\mathcal{D}^{i+1}\right) \subset \mathcal{D}^{i+1}$.

Now let $D \in \mathcal{D}^{1}: \Psi(D) \in \mathcal{D}^{1}$. Since for any $f \in \mathcal{A}$,

$$
2 \Psi(f[D, f])=\Psi\left(\left[D, f^{2}\right]\right)=\left[\Psi(D), f^{2}\right]=2 f[\Psi(D), f]=2 f \Psi([D, f]),
$$

we have

$$
\Psi(f \hat{D}(f))=f \Psi(\hat{D}(f)),
$$




\section{Automorphisms of Quantum and Classical Poisson algebras}

for any $f \in \mathcal{A}, D \in \mathcal{D}^{1}$. Substituting $D:=g D\left(g \in \mathcal{A}, D \in \mathcal{D}^{1}\right)$ and $f:=f+h(f, h \in \mathcal{A})$ in $(5)$, we get

$$
\Psi(f g \hat{D}(h))+\Psi(g h \hat{D}(f))=f \Psi(g \hat{D}(h))+h \Psi(g \hat{D}(f)) .
$$

For $g=\hat{D}(h)$, Equation (6) reads

$$
\Psi\left(f(\hat{D}(h))^{2}\right)+\Psi(h \hat{D}(f) \hat{D}(h))=f \Psi\left((\hat{D}(h))^{2}\right)+h \Psi(\hat{D}(f) \hat{D}(h)),
$$

where the last terms of the left-hand side and the right-hand side cancel in view of (5) applied to $D:=\hat{D}(f) D$. Hence, for each $f, h \in \mathcal{A}$ and $D \in \mathcal{D}^{1}$,

$$
\Psi\left(f(\hat{D}(h))^{2}\right)=f \Psi\left((\hat{D}(h))^{2}\right) .
$$

The last equation shows that the radical $\operatorname{rad}(J)$ of the ideal $J=\{g \in \mathcal{A}: \Psi(f g)=f \Psi(g), \forall f \in \mathcal{A}\}$ of the associative commutative algebra $\mathcal{A}$, contains $\left[\mathcal{D}^{1}, \mathcal{A}\right]$. Since $\mathcal{D}$ is non-singular, this implies that $J=\mathcal{A}$, so that $\Psi(f)=\Psi(1) f$, for all $f \in \mathcal{A}$. It is obvious that $\Psi_{1}=\Psi-m_{\Psi(1)}$ belongs to $C(\mathcal{D})$ and respects the filtration, and we easily see that it is lowering. Indeed, since $\Psi_{1}(\mathcal{A})=0$, assume inductively that $\Psi_{1}\left(\mathcal{D}^{i}\right) \subset \mathcal{D}^{i-1}$. Then, $\left[\Psi_{1}\left(\mathcal{D}^{i+1}\right), \mathcal{A}\right]=\Psi_{1}\left(\left[\mathcal{D}^{i+1}, \mathcal{A}\right]\right) \subset \mathcal{D}^{i-1}$ and $\Psi_{1}\left(\mathcal{D}^{i+1}\right) \subset \mathcal{D}^{i}$.

Theorem 4. Let $\mathcal{D}_{i}$ be distinguishing, non-singular and symplectic, $i=1,2$. Then every isomorphism $\Phi: \mathcal{D}_{1} \rightarrow \mathcal{D}_{2}$ of the Lie algebras $\left(\mathcal{D}_{i},[\cdot, \cdot]\right), i=1,2$, respects the filtration and its restriction $\left.\Phi\right|_{\mathcal{A}_{1}}$ to $\mathcal{A}_{1}$ has the form

$$
\left.\Phi\right|_{\mathcal{A}_{1}}=\kappa A,
$$

where $\kappa \in K, \kappa \neq 0$ and $A: \mathcal{A}_{1} \rightarrow \mathcal{A}_{2}$ is an isomorphism of the associative commutative algebras. The same is true for any isomorphism $\Phi: \mathcal{D}_{1}^{1} \rightarrow \mathcal{D}_{2}^{1}$ of the corresponding Lie algebras of first-order differential operators.

Proof. By Corollary 2, $\Phi$ respects the filtration, so $\Phi\left(\mathcal{A}_{1}\right)=\mathcal{A}_{2}$. Let

$$
\Phi_{*}: \operatorname{Hom}_{K}\left(\mathcal{D}_{1}, \mathcal{D}_{1}\right) \rightarrow \operatorname{Hom}_{K}\left(\mathcal{D}_{2}, \mathcal{D}_{2}\right)
$$

be the induced isomorphism of the Lie algebras of linear homomorphisms, defined for $\Psi \in \operatorname{Hom}_{K}\left(\mathcal{D}_{1}, \mathcal{D}_{1}\right)$ by

$$
\Phi_{*}(\Psi)=\Phi \circ \Psi \circ \Phi^{-1} .
$$

Since $\Phi\left(\mathcal{A}_{1}\right)=\mathcal{A}_{2}, \Phi_{*}\left(C\left(\mathcal{D}_{1}\right)\right)=C\left(\mathcal{D}_{2}\right)$; in particular, $\Phi_{*}\left(m_{g}\right) \in C\left(\mathcal{D}_{2}\right)$ for $g \in \mathcal{A}_{1}$. By Theorem 3 ,

$$
\Phi_{*}\left(m_{g}\right)\left(f^{\prime}\right)=\Phi_{*}\left(m_{g}\right)(1) \cdot f^{\prime},
$$

i.e.

$$
\Phi\left(g \cdot \Phi^{-1}\left(f^{\prime}\right)\right)=\Phi\left(g \cdot \Phi^{-1}(1)\right) \cdot f^{\prime},
$$

for all $f^{\prime} \in \mathcal{A}_{2}$. Observe that $\Phi^{-1}(1)$ is central in $\mathcal{D}_{1}$ and is thus a non-vanishing constant $\kappa^{-1}$. Substituting $\Phi(f)\left(f \in \mathcal{A}_{1}\right)$ for $f^{\prime}$ in $(7)$, we obtain $\Phi(f \cdot g)=\kappa^{-1} \Phi(f) \cdot \Phi(g)$. For $A$ defined by $A(f)=\kappa^{-1} \Phi(f)$, this reads $A(f \cdot g)=A(f) \cdot A(g)$, which completes the proof of Theorem 4 .

We can prove in the same way - mutatis mutandis - that Theorem 4 is still valid for $\mathcal{D}_{i}, i=1,2$, replaced by classical Poisson algebras $S_{i}, i=1,2$.

Theorem 5. Let $S_{i}$ be a distinguishing, non-singular and symplectic classical Poisson algebra, $i=1,2$. Then every isomorphism $\Phi: S_{1} \rightarrow S_{2}$ of the Lie algebras $\left(S_{i},\{\cdot, \cdot\}\right), i=1,2$, respects the filtration and its restriction $\left.\Phi\right|_{\mathcal{A}_{1}}$ to $\mathcal{A}_{1}$ has the form

$$
\left.\Phi\right|_{\mathcal{A}_{1}}=\kappa A,
$$

where $\kappa \in K, \kappa \neq 0$ and $A: \mathcal{A}_{1} \rightarrow \mathcal{A}_{2}$ is an isomorphism of the associative commutative algebras. 


\section{J. Grabowski And N. Poncin}

COROllary 3. If two distinguishing, non-singular and symplectic quantum (respectively classical) Poisson algebras are isomorphic as Lie algebras, then their basic algebras are isomorphic as associative algebras. The same remains true for Lie subalgebras of the first-order operators of such Poisson algebras: if they are isomorphic, then their basic algebras are isomorphic associative algebras.

Let us now return to the quantum Poisson algebra $\mathcal{D}=\mathcal{D}(M)$ of differential operators of a smooth, Hausdorff, second countable, connected manifold $M$. It is well known that every associative algebra isomorphism $A: \mathcal{A}_{1}=C^{\infty}\left(M_{1}\right) \rightarrow \mathcal{A}_{2}=C^{\infty}\left(M_{2}\right)$ is of the form

$$
A: \mathcal{A}_{1} \ni f \rightarrow f \circ \phi^{-1} \in \mathcal{A}_{2},
$$

where $\phi: M_{1} \rightarrow M_{2}$ is a diffeomorphism. Thus, we can draw a conclusion of the same type as a classical result of Pursell and Shanks [PS54] (see also [Gra78]).

TheOREM 6. The Lie algebras $\mathcal{D}\left(M_{1}\right)$ and $\mathcal{D}\left(M_{2}\right)$ (respectively $\mathcal{D}^{1}\left(M_{1}\right)$ and $\mathcal{D}^{1}\left(M_{2}\right)$, or $S\left(M_{1}\right)$ and $S\left(M_{2}\right)$ ) of all differential operators (respectively all differential operators of order 1, or all symmetric contravariant tensors) on two smooth manifolds $M_{1}$ and $M_{2}$ are isomorphic if and only if the manifolds $M_{1}$ and $M_{2}$ are diffeomorphic.

Studying the isomorphisms mentioned in the above theorem reduces then to studying the automorphisms of the Lie algebras $\mathcal{D}(M), \mathcal{D}^{1}(M)$ and $S(M)$.

\section{Particular automorphisms}

In the following, $\mathcal{D}$ denotes the quantum algebra $\mathcal{D}(M)$ and $S$ is its classical limit $S(M)$.

\section{1}

Every automorphism $A$ of the associative algebra $\mathcal{A}=C^{\infty}(M)$ (which is implemented by a diffeomorphism $\phi$ of $M$ ) induces an automorphism $A_{*}$ of the Lie algebra $\mathcal{D}$ :

$$
A_{*}(D)=A \circ D \circ A^{-1} \quad(D \in \mathcal{D}) .
$$

It clearly restricts to an automorphism of $\mathcal{D}^{1}$. The automorphism $A$ also induces an automorphism $A_{*}$ of $S$. It is simply induced by the phase lift of the diffeomorphism $\phi$ to the cotangent bundle $T^{*} M$ if we interpret elements of $S$ as polynomial functions on $T^{*} M$. If we interpret $S$ as symmetric contravariant tensors on $M$, then $A_{*}$ is simply the action of $\phi$ on such tensors.

Now let $\Phi \in \operatorname{Aut}(\mathcal{D},[\cdot, \cdot])$ (respectively $\Phi \in \operatorname{Aut}\left(\mathcal{D}^{1},[\cdot, \cdot]\right)$ or $\left.\Phi \in \operatorname{Aut}(S,\{\cdot, \cdot\})\right)$. By Theorem 4, there are $A \in \operatorname{Aut}(\mathcal{A}, \cdot)$ and $\kappa \in K, \kappa \neq 0$, such that $\left.\Phi\right|_{\mathcal{A}}=\left.\kappa A_{*}\right|_{\mathcal{A}}$. Then, $\Phi_{1}=\left(A_{*}\right)^{-1} \circ \Phi$ is an automorphism of $\mathcal{D}$ (respectively $\mathcal{D}^{1}$ or $S$ ), which is $\kappa \cdot$ id (id is the identity map) on $\mathcal{A}$. It is thus sufficient to describe the automorphisms that are $\kappa \cdot$ id on functions.

\section{2}

Let $\omega \in \Omega^{1}(M) \cap \operatorname{ker} d$ be a closed 1-form on $M$ and $D \in \mathcal{D}^{i}$. If $U$ is an open subset of $M$ and $\left.\omega\right|_{U}=d\left(f_{U}\right)\left(f_{U} \in C^{\infty}(U)\right)$, the operators $\left[\left.D\right|_{U}, f_{U}\right] \in \mathcal{D}_{U}^{i-1}\left(\mathcal{D}_{U}^{k}\right.$ is defined as $\mathcal{D}^{k}$ but for $\left.M=U\right)$ are of course the restrictions of a unique well-defined operator $\bar{\omega}(D) \in \mathcal{D}^{i-1}$ :

$$
\left.\bar{\omega}(D)\right|_{U}=\left[\left.D\right|_{U}, f_{U}\right]
$$

since the above commutator does not depend on the choice of $f_{U}$ with $\left.\omega\right|_{U}=d\left(f_{U}\right)$ (constants are central with respect to the bracket). It is clear that $\bar{\omega} \in \mathcal{L}(\mathcal{D}, \mathcal{D}) \cap \mathcal{L}\left(\mathcal{D}^{i}, \mathcal{D}^{i-1}\right)$, that $\bar{\omega}(X)=$ $\omega(X)$ for all $X \in \operatorname{Vect}(M)$, and that $\omega \mapsto \bar{\omega}$ is linear. Moreover, $\bar{\omega}$ is a 1 -cocycle of the adjoint Chevalley-Eilenberg cohomology of $\mathcal{D}$, i.e. a derivation of $\mathcal{D}$. Since $\bar{\omega}$ is lowering, it is locally 


\section{Automorphisms of QUANTUm AND Classical Poisson Algebras}

nilpotent, so that

$$
e^{\bar{\omega}}=\mathrm{id}+\bar{\omega}+\frac{1}{2 !} \bar{\omega}^{2}+\cdots
$$

is well defined and is an automorphism of $\mathcal{D}$ (that is the identity on functions). In particular, for $\omega=d f$, the automorphism $e^{\bar{\omega}}$ is just the inner automorphism $\mathcal{D} \ni D \mapsto e^{f} \cdot D \cdot e^{-f} \in \mathcal{D}$.

On the classical level, we have an analogous derivation of the classical Poisson algebra $S$ :

$$
\left.\bar{\omega}(P)\right|_{U}=\left\{\left.P\right|_{U}, f_{U}\right\},
$$

and the analogous automorphism $e^{\bar{\omega}}$. These automorphisms have a geometric description, if we interpret $S$ as the Lie algebra of polynomial functions on the cotangent bundle $T^{*} M$ with the canonical Poisson bracket. Every closed 1-form $\omega$ on $M$ induces a vertical locally Hamiltonian vector field $\omega^{\mathrm{v}}$ on $T^{*} M$ which connects the 0 -section of $T^{*} M$ with another Lagrangian submanifold which is the image of the section $\omega$. If, locally, $\omega=d f$, then $\omega^{\mathrm{v}}$ is, locally, the Hamiltonian vector field of the pull-back of $f$ to $T^{*} M$. In the pure vector bundle language, $\omega^{\mathrm{v}}$ is simply the vertical lift of the section $\omega$ of $T^{*} M$. Since this vector field is vertical and constant on fibers, it is complete and determines a one-parameter group $\operatorname{Exp}\left(\omega^{\mathrm{v}}\right)$ of symplectomorphisms of $T^{*} M$. The automorphism $e^{\bar{\omega}}$ is just the action of $\operatorname{Exp}\left(\omega^{\mathrm{v}}\right)$ on polynomial functions on $T^{*} M$. The symplectomorphism $\operatorname{Exp}\left(\omega^{\mathrm{v}}\right)$ translates every covector $\eta_{p}$ to $\eta_{p}+\omega(p)$.

\section{3}

The following remark concerns the divergence operator on an arbitrary manifold $M$. For further details the reader is referred to [Lec02].

Denote by $\mathbb{F}_{\lambda}(T M)(\lambda \in \mathbb{R})$ the vector bundle (of rank 1) of $\lambda$-densities and by $\mathcal{F}_{\lambda}(M)$ the $\operatorname{Vect}(M)$-module of $\lambda$-density fields (or simply $\lambda$-densities) on $M$ (i.e. the space of smooth sections of $\mathbb{F}_{\lambda}(T M)$, endowed with the natural Lie derivative $L_{X}, X \in \operatorname{Vect}(M)$ ). The result stating that these modules $\mathcal{F}_{\lambda}(M)$ are not isomorphic, implies the existence of a non-trivial 1-cocycle of the Lie algebra $\operatorname{Vect}(M)$ canonically represented on $C^{\infty}(M)$. It appears if $\mathcal{F}_{\lambda}(M)$ is viewed as a deformation of $\mathcal{F}_{0}(M)=C^{\infty}(M)$.

Let us be somewhat more precise. In the proof of triviality of the bundles $\mathbb{F}_{\lambda}(T M)$, we construct a section that is everywhere non-zero (and even, which has at each point only strictly positive values). Let $\rho_{0} \in \mathcal{F}_{1}(M)$ be such a section. Then $\rho_{0}^{\lambda} \in \mathcal{F}_{\lambda}(M)$ also vanishes nowhere and $\tau_{0}^{\lambda}: f \in$ $C^{\infty}(M) \longrightarrow f \rho_{0}^{\lambda} \in \mathcal{F}_{\lambda}(M)$ is a bijection. We have the subsequent results:

- there is a 1-cocycle $\gamma: \operatorname{Vect}(M) \longrightarrow C^{\infty}(M)$, which depends on $\rho_{0}$ but not on $\lambda$, such that, for any $X \in \operatorname{Vect}(M)$,

$$
\left(\tau_{0}^{\lambda}\right)^{-1} \circ L_{X} \circ \tau_{0}^{\lambda}: f \in C^{\infty}(M) \longrightarrow X(f)+\lambda \gamma(X) f \in C^{\infty}(M) ;
$$

- the cocycle $\gamma$ is a differential operator with symbol $\sigma(\gamma)(\zeta ; X)=\langle X, \zeta\rangle$, where $\langle X, \zeta\rangle$ denotes the evaluation of $\zeta \in T_{x}^{*} M$ upon $X \in T_{x} M$;

- the cohomology class of $\gamma$ is independent of $\rho_{0}$.

This class $\operatorname{div}_{M}$ is the class of the divergence. Each cocycle cohomologous to $\gamma$ will be called a divergence. Finally, the following propositions hold.

- The first cohomology space of $\operatorname{Vect}(M)$ represented upon $C^{\infty}(M)$ is given by

$$
H^{1}\left(\operatorname{Vect}(M), C^{\infty}(M)\right)=\mathbb{R} \operatorname{div}_{M} \oplus H_{\mathrm{DR}}^{1}(M),
$$

where $H_{\mathrm{DR}}^{1}(M)$ denotes the first space of the de Rham cohomology of $M$. 


\section{J. Grabowski and N. Poncin}

- For any divergence $\gamma$ on $M$, there is an atlas of $M$, such that in every chart,

$$
\gamma(X)=\sum_{i} \partial_{x^{i}} X^{i}, \quad \text { for all } X \in \operatorname{Vect}(M),
$$

with self-explaining notation.

The preceding results have a simple explanation. Remember that if the manifold $M$ is orientable and if $\Omega$ is a fixed volume form, the divergence of $X \in \operatorname{Vect}(M)$ with respect to $\Omega$ is defined as the smooth function $\operatorname{div}_{\Omega} X$ of $M$ that verifies $L_{X} \Omega=\left(\operatorname{div}_{\Omega} X\right) \Omega$. One easily sees that $\operatorname{div}_{-\Omega} X=$ $\operatorname{div}_{\Omega} X$. However, this means that the divergence of a vector field can even be defined on a nonorientable manifold with respect to a pseudo-volume form.

The divergence operator associated to a 1-density $\rho_{0}$, will be denoted by $\operatorname{div}_{\rho_{0}}$ or simply div, if no confusion is possible. Let us fix a divergence on $\operatorname{Vect}(M)$.

Lemma 1. There is a unique $\mathcal{C} \in \operatorname{Aut}(\mathcal{D},[\cdot, \cdot])$, such that $\mathcal{C}(f)=-f, \mathcal{C}(X)=X+\operatorname{div} X$,

$$
\mathcal{C}(D \circ f)=f \circ \mathcal{C}(D),
$$

and

$$
\mathcal{C}(D \circ X)=-\mathcal{C}(X) \circ \mathcal{C}(D)
$$

for all $f \in \mathcal{A}, X \in \mathcal{D}_{c}^{1}$, and $D \in \mathcal{D}$.

Proof. Consider an atlas of $M$, such that the divergence has the form (9) in any chart. Then, in every chart $\left(U,\left(x^{1}, \ldots, x^{n}\right)\right), \mathcal{C}$ which is given by $\mathcal{C}\left(\eta ; P_{k}\right)(\xi)=(-1)^{k+1} P_{k}(\xi+\eta)$, where $P_{k} \in \bigvee^{k} \mathbb{R}^{n}$ is a homogeneous polynomial of degree $k$, defines an operator $\mathcal{C}_{U}: \mathcal{D}_{U} \longrightarrow \mathcal{D}_{U}$ that (maps $\mathcal{D}_{U}^{i}$ into $\mathcal{D}_{U}^{i}$ and) verifies the above characteristic properties. Let us explain for instance the fourth property; the third is analogous and the first and second are obvious. Use the previously mentioned simplifications of notations, identify the space $\mathcal{D}_{U}^{i}$ of differential operators to the space $C^{\infty}\left(U, \bigvee^{\leqslant i} \mathbb{R}^{n}\right)$ of polynomial representations, set $X=g X$ and $D=h P_{k}$ (on the left-hand side $X \in C^{\infty}\left(U, \mathbb{R}^{n}\right)$ and $D \in C^{\infty}\left(U, \bigvee^{\leqslant i} \mathbb{R}^{n}\right)$, on the right-hand side $g, h \in C^{\infty}(U), X \in \mathbb{R}^{n}$, and $\left.P_{k} \in \bigvee^{k} \mathbb{R}^{n}(k \leqslant i)\right)$ and symbolize the derivatives acting on $g, h$ and the argument $f \in C^{\infty}(U)$ of $D \circ X, \mathcal{C}_{U}(D \circ X)$ and $\mathcal{C}_{U}(X) \circ \mathcal{C}_{U}(D)$, by $\zeta, \eta$ and $\xi$, respectively. Since

$$
(D \circ X)(f)=D(X(f)) \simeq\langle X, \xi\rangle P_{k}(\xi+\zeta)=\langle X, \xi\rangle \sum_{\ell} \frac{1}{\ell !}\left(\zeta \partial_{\xi}\right)^{\ell} P_{k}(\xi)
$$

$\left(\zeta \partial_{\xi}\right.$ is the derivative with respect to $\xi$ in the direction of $\zeta$ ), we have

$$
\begin{aligned}
\left(\mathcal{C}_{U}(D \circ X)\right)(f) & \simeq \sum_{\ell} \frac{1}{\ell !} \mathcal{C}\left(\eta+\zeta ; X\left(\zeta \partial_{\xi}\right)^{\ell} P_{k}\right)(\xi) \\
& =(-1)^{k}\langle X, \xi+\eta+\zeta\rangle\left(\sum_{\ell} \frac{1}{\ell !}\left((-\zeta) \partial_{\xi}\right)^{\ell} P_{k}\right)(\xi+\eta+\zeta) \\
& =(-1)^{k}\langle X, \xi+\eta+\zeta\rangle P_{k}(\xi+\eta) \\
& \simeq-\left(\mathcal{C}_{U}(X) \circ \mathcal{C}_{U}(D)\right)(f) .
\end{aligned}
$$

It is well known that any differential operator $D \in \mathcal{D}$ has a global (not necessarily unique) decomposition as a finite sum of terms of the type $f X_{k} \circ \cdots \circ X_{1}\left(f \in C^{\infty}(M), X_{\ell} \in \operatorname{Vect}(M)\right)$. If we set $\mathcal{L}_{X}^{1}=X+\operatorname{div} X(X \in \operatorname{Vect}(M))$, we have $\mathcal{C}_{U}\left(\left.X\right|_{U}\right)=\left.\mathcal{L}_{X}^{1}\right|_{U}$ and $\mathcal{C}_{U}\left(\left.D\right|_{U}\right)=\left((-1)^{k+1} \mathcal{L}_{X_{1}}^{1}\right.$ 。 $\left.\cdots \circ \mathcal{L}_{X_{k}}^{1} \circ f\right)\left.\right|_{U}$. This means that the $\mathcal{C}_{U}$ are the restrictions of a unique well-defined operator

$$
\mathcal{C}: \mathcal{D} \ni D=f X_{k} \circ \cdots \circ X_{1} \longrightarrow \mathcal{C}(D)=(-1)^{k+1} \mathcal{L}_{X_{1}}^{1} \circ \cdots \circ \mathcal{L}_{X_{k}}^{1} \circ f \in \mathcal{D},
$$

which inherits the characteristic properties. 


\section{Automorphisms of QUANTUm AND Classical Poisson Algebras}

The homomorphism property, $\mathcal{C}[D, \Delta]=[\mathcal{C} D, \mathcal{C} \Delta](D, \Delta \in \mathcal{D})$, is a direct consequence of the characteristic properties and the definition of $\mathcal{C}$. Noting that $\mathcal{C}^{2}(X)=X$ and, from the preceding verification, that $\mathcal{C}(D \circ \Delta)=-\mathcal{C}(\Delta) \circ \mathcal{C}(D)$, we immediately see that $\mathcal{C}^{2}=$ id, so $\mathcal{C} \in \operatorname{Aut}(\mathcal{D})$.

Remark 1. We easily convince ourselves that, if $\Omega$ is a volume form of $M, \mathcal{C}$ is the opposite of the conjugation $*: \mathcal{D} \ni D \longrightarrow D^{*} \in \mathcal{D}$ of differential operators, defined by

$$
\int_{M} D(f) \cdot g|\Omega|=\int_{M} f \cdot D^{*}(g)|\Omega|,
$$

for all compactly supported $f, g \in C^{\infty}(M)$.

\section{4}

On $S$, as on every graded algebra, there is a canonical one-parameter family of automorphisms $U_{\kappa}$, $\kappa \neq 0$, namely $U_{\kappa}(P)=\kappa^{1-i} P$ for $P \in S_{i}$. It is easy to see that $U_{\kappa}$ is an automorphism of the Lie algebra $S$. For positive $\kappa$, this is the one-parameter group of automorphisms induced by the canonical derivation Deg : $S \rightarrow S$ of the Poisson bracket, $\operatorname{Deg}(P)=(i-1) P$ for $P \in S_{i}$, namely $U_{\kappa}=$ $e^{-\log (\kappa) \operatorname{Deg}}$. Since $\left.U_{\kappa}\right|_{\mathcal{A}}=\left.\kappa \cdot \mathrm{id}\right|_{\mathcal{A}}$, we can now reduce every automorphism $\Phi$ of the Lie algebra $S$ to the case when $\left.\Phi\right|_{\mathcal{A}}=\left.\mathrm{id}\right|_{\mathcal{A}}$.

\section{Automorphisms of the Lie algebra $\mathcal{D}^{1}(M)$}

When using the decomposition $\mathcal{D}=\mathcal{A} \oplus \mathcal{D}_{c}$, we denote by $\pi_{0}$ and $\pi_{c}$ the projections onto $\mathcal{A}$ and $\mathcal{D}_{c}$, respectively. Furthermore, if $D \in \mathcal{D}$, we set $D_{0}=\pi_{0} D=D(1)$ and $D_{c}=\pi_{c} D=D-D(1)$ and if $\Phi \in \mathcal{L}(\mathcal{D})$, we set $\Phi_{0}=\pi_{0} \circ \Phi \in \mathcal{L}(\mathcal{D}, \mathcal{A})$ and $\Phi_{c}=\pi_{c} \circ \Phi \in \mathcal{L}\left(\mathcal{D}, \mathcal{D}_{c}\right)$. Note also that for $f, g \in \mathcal{A}$, we have $\left[D_{c}, f\right]_{0}=D_{c}(f)$ and $\left[D_{c}, f\right]_{c}(g)=D_{c}(f \cdot g)-D_{c}(f) \cdot g-f \cdot D_{c}(g)$, so that $\left[D_{c}, f\right]_{0}=0$, for all $f \in \mathcal{A}$ if and only if $D_{c}=0$ and $\left[D_{c}, f\right]_{c}(g)=0$, for all $f, g \in \mathcal{A}$ if and only if $D_{c} \in \mathcal{D}_{c}^{1}$.

Let us now return to the problem of the determination of all automorphisms $\Phi$ of $\mathcal{D}$ (respectively $\mathcal{D}^{1}$ ) that coincide with $\kappa \cdot$ id on functions.

The projection of the homomorphism property, written for $D_{c} \in \mathcal{D}_{c}$ and $f \in \mathcal{A}$, leads to the equations

$$
\left(\Phi_{c} D_{c}\right)(f)=\kappa^{-1} \Phi_{0}\left[D_{c}, f\right]=D_{c}(f)+\kappa^{-1} \Phi_{0}\left[D_{c}, f\right]_{c}
$$

and

$$
\left[\Phi_{c} D_{c}, f\right]_{c}=\kappa^{-1} \Phi_{c}\left[D_{c}, f\right]_{c}
$$

and its projection, if it is written for $D_{c}, \Delta_{c} \in \mathcal{D}_{c}$, gives

$$
\Phi_{0}\left[D_{c}, \Delta_{c}\right]=\left(\Phi_{c} D_{c}\right)\left(\Phi_{0} \Delta_{c}\right)-\left(\Phi_{c} \Delta_{c}\right)\left(\Phi_{0} D_{c}\right)
$$

and

$$
\Phi_{c}\left[D_{c}, \Delta_{c}\right]=\left[\Phi_{c} D_{c}, \Phi_{0} \Delta_{c}\right]_{c}+\left[\Phi_{0} D_{c}, \Phi_{c} \Delta_{c}\right]_{c}+\left[\Phi_{c} D_{c}, \Phi_{c} \Delta_{c}\right]
$$

If we write these equations for $D_{c}$ and $\Delta_{c}$ in the Lie subalgebra $\mathcal{D}_{c}^{1}$ of $\mathcal{D}_{c}$, (10) means that $\left.\Phi_{c}\right|_{\mathcal{D}_{c}^{1}}=\mathrm{id},(11)$ and (13) are trivial and (12) tells us that $\alpha:=\left.\Phi_{0}\right|_{\mathcal{D}_{c}^{1}}$ is a 1-cocycle of the Lie algebra of vector fields canonically represented on functions by the Lie derivative. As, in view of (8),

$$
\alpha=\lambda \operatorname{div}+\omega \quad\left(\lambda \in \mathbb{R}, \omega \in \Omega^{1}(M) \cap \operatorname{ker} d\right),
$$

we have the following.

Theorem 7. The automorphisms $\Phi_{1}$ of $\mathcal{D}^{1}(M)$ that verify $\left.\Phi_{1}\right|_{C^{\infty}(M)}=\kappa \cdot$ id $(\kappa \in \mathbb{R}, \kappa \neq 0)$ are the mappings

$$
\Phi_{1}=\kappa \pi_{0}+(\mathrm{id}+\lambda \operatorname{div}+\omega) \circ \pi_{c},
$$

where $\lambda \in \mathbb{R}$ and $\omega \in \Omega^{1}(M) \cap \operatorname{ker} d$. 


\section{J. Grabowski And N. Poncin}

Indeed, one easily sees that these homomorphisms of $\mathcal{D}^{1}$ are bijective. We can now summarize all facts and give the complete description of automorphisms of $\mathcal{D}^{1}$.

Theorem 8. A linear map $\Phi: \mathcal{D}^{1}(M) \rightarrow \mathcal{D}^{1}(M)$ is an automorphism of the Lie algebra $\mathcal{D}^{1}(M)=$ $\operatorname{Vect}(M) \oplus C^{\infty}(M)$ of linear first-order differential operators on $C^{\infty}(M)$ if and only if it can be written in the form

$$
\Phi(X+f)=\phi_{*}(X)+(\kappa f+\lambda \operatorname{div} X+\omega(X)) \circ \phi^{-1},
$$

where $\phi$ is a diffeomorphism of $M, \lambda, \kappa$ are constants, $\kappa \neq 0, \omega$ is a closed 1-form on $M$ and $\phi_{*}$ is defined by

$$
\left(\phi_{*}(X)\right)(f)=(X(f \circ \phi)) \circ \phi^{-1} .
$$

All the objects $\phi, \lambda, \kappa, \omega$ are uniquely determined by $\Phi$.

\section{Automorphisms of the Lie algebra $S(M)$}

We now finish the description of automorphisms of the Lie algebra $S(M)$. We have already reduced the problem to automorphisms which are identity on $\mathcal{A}=C^{\infty}(M)$. Such an automorphism, respecting the filtration, restricts to an automorphism of $\mathcal{D}^{1}(M)=S^{1}(M)$, where, in view of Theorem 8 , it is of the form $\Phi(X+f)=X+(f+\lambda \operatorname{div} X+\omega(X))$. Using the automorphism $e^{\bar{\omega}}$, we can reduce to the case when $\omega=0$. We show that in this case $\lambda=0$ and $\Phi=\mathrm{id}$.

Consider an automorphism $\Phi$ of $S$ which is identical on functions and of the form $\Phi(X)=$ $X+\lambda \operatorname{div} X$ on vector fields. It is easy to see that this implies that $\Phi=\operatorname{id}_{S}+\psi$, where $\psi: S \rightarrow S$ is lowering. The automorphism property yields $\psi(\{P, f\})=\{\psi(P), f\}$, for all $P \in S, f \in \mathcal{A}$. Let us take $P \in S_{2}$. Then $\{P, f\}$ is a vector field (linear function on $T^{*} M$ ) and we get $\lambda \operatorname{div}\{P, f\}=$ $\{\psi(P), f\}$. However, for $\lambda \neq 0$, the left-hand side is a second-order differential operator with respect

to $f$ (e.g. for $P=X^{2}$, the principal symbol is $2 \lambda X^{2}$ ), while the right-hand side is of first order - a contradiction. Thus $\lambda=0$ and $\Phi$ is identity on first-order operators (polynomials).

Now we can proceed inductively, showing that $\left.\psi\right|_{S_{i}}=0$ also for $i>1$. For any $P \in S$, we have

$$
\{\psi(P), f\}=\psi(\{P, f\}) \text { and }\{\psi(P), X\}=\psi(\{P, X\}),
$$

for any function $f$ and any vector field $X$. Then, $\left.\psi\right|_{S_{i-1}}=0$ and (15) imply that, for $P \in S_{i}$, we have $\psi(P) \in \mathcal{A}$ and that $\psi$ is an intertwining operator for the action of vector fields on $S_{i}$ and $\mathcal{A}$. However, following the methods of [Pon02] or [BHMP02], one can easily see that such operators are trivial, so $\left.\psi\right|_{S_{i}}=0$. Thus we get $\psi=0$, i.e. $\Phi=\mathrm{id}_{S}$, and we can formulate the following final result.

Theorem 9. A linear map $\Phi: S(M) \rightarrow S(M)$ is an automorphism of the Lie algebra $S(M)$ of polynomial functions on $T^{*} M$ with respect to the canonical symplectic bracket if and only if it can be written in the form

$$
\Phi(P)=U_{\kappa}(P) \circ \phi^{*} \circ \operatorname{Exp}\left(\omega^{\mathrm{v}}\right)
$$

where $\kappa$ is a non-zero constant, $U_{\kappa}(P)=\kappa^{1-i} P$ for $P \in S_{i}, \phi^{*}$ is the phase lift of a diffeomorphism $\phi$ of $M$ and $\operatorname{Exp}\left(\omega^{\mathrm{v}}\right)$ is the vertical symplectic diffeomorphism of $T^{*} M$ being the translation by a closed 1-form $\omega$ on $M$. All the objects $\kappa, \phi, \omega$ are uniquely determined by $\Phi$.

The automorphisms of the whole Poisson algebra $C^{\infty}(N)$ on a symplectic (or even a Poisson) manifold $N$, have been described in [AG90, Gra00]. Our symplectic manifold is particular here (e.g. $N=T^{*} M$ is non-compact and the symplectic form is exact), so the result of [AG90] states that automorphisms of the Poisson algebra $C^{\infty}\left(T^{*} M\right)$ are of the form $P \mapsto s P \circ \tilde{\phi}$, where $s$ is a non-zero constant and $\tilde{\phi}$ is a conformal symplectomorphism with the conformal constant $s$. In our case, we deal with a subalgebra of polynomial functions which is only preserved by two types of conformal 


\section{Automorphisms of Quantum and Classical Poisson algebras}

symplectomorphisms: phase lifts of diffeomorphisms of $M$ and vertical symplectomorphisms associated with closed 1-forms on $M$. In both cases we have symplectomorphisms, so $s=1$. So far so good, the pictures coincide, but for $S$ we get an additional family of automorphisms $U_{\kappa}$. These automorphisms simply do not extend to automorphisms of the whole algebra $C^{\infty}\left(T^{*} M\right)$.

\section{Automorphisms of the Lie algebra $\mathcal{D}(M)$}

Let us go back to the general problem of the determination of the automorphisms $\Phi_{1}$ of $\mathcal{D}=\mathcal{D}(M)$, with restriction $\kappa \cdot$ id $(\kappa \in \mathbb{R}, \kappa \neq 0)$ on $\mathcal{A}=C^{\infty}(M)$.

The restriction $\left.\Phi_{1}\right|_{\mathcal{D}^{1}}$ has the form given by Theorem 7 . When setting

$$
\Phi_{2}=\Phi_{1} \circ e^{-\kappa^{-1} \bar{\omega}},
$$

we obtain - as easily verified - an automorphism of $\mathcal{D}$, whose restriction to $\mathcal{D}^{1}$ is

$$
\left.\Phi_{2}\right|_{\mathcal{D}^{1}}=\left.\left(\kappa \pi_{0}+(\mathrm{id}+\beta) \circ \pi_{c}\right)\right|_{\mathcal{D}^{1}}, \quad \text { where } \beta=\lambda \operatorname{div} .
$$

In the following, we write $\Phi$ instead of $\Phi_{2}$ (if no confusion is possible). Using (11), we find that

$$
\left[\left[\left[\Phi_{c} D_{c}^{i}, f_{1}\right]_{c}, f_{2}\right]_{c}, \ldots, f_{i-1}\right]_{c}=\kappa^{1-i}\left[\left[\left[D_{c}^{i}, f_{1}\right]_{c}, f_{2}\right]_{c}, \ldots, f_{i-1}\right]_{c},
$$

since $\left.\Phi_{c}\right|_{\mathcal{D}_{c}^{1}}=$ id. So $\Phi_{c} D_{c}^{i}-\kappa^{1-i} D_{c}^{i} \in \mathcal{D}_{c}^{i-1}$ and

$$
\left.\Phi\right|_{\mathcal{D}^{i}}=\kappa^{1-i} \mathrm{id}+\psi_{i}
$$

with $\psi_{i} \in \mathcal{L}\left(\mathcal{D}^{i}, \mathcal{D}^{i-1}\right)$. Note that $\psi_{0}=0, \psi_{1}=\left.\left((\kappa-1) \pi_{0}+\beta \circ \pi_{c}\right)\right|_{\mathcal{D}^{1}}$ and $\psi_{i} f=\kappa\left(1-\kappa^{-i}\right) f$.

Remark 2. Assertion (18) is equivalent to saying that the automorphism $\tilde{\Phi}$ of the Poisson algebra $S$ induced by $\Phi$ is $U_{\kappa}$ (cf. Theorem 9 ).

Now apply (18), observe that the homomorphism property then reads

$$
\psi_{i+j-1}\left[D^{i}, \Delta^{j}\right]=\kappa^{1-j}\left[\psi_{i} D^{i}, \Delta^{j}\right]+\kappa^{1-i}\left[D^{i}, \psi_{j} \Delta^{j}\right]+\left[\psi_{i} D^{i}, \psi_{j} \Delta^{j}\right],
$$

for all $D^{i} \in \mathcal{D}^{i}, \Delta^{j} \in \mathcal{D}^{j}$ and project (19), written for $D_{c}^{i}$ and $f(i \geqslant 2)$ and for $D_{c}^{i}$ and $\Delta_{c}^{j}(i+j \geqslant 3)$, on $\mathcal{A}$ :

$$
\left(\psi_{i, c} D_{c}^{i}\right)(f)=\kappa^{-1} \psi_{i-1,0}\left[D_{c}^{i}, f\right]=\left(1-\kappa^{1-i}\right) D_{c}^{i}(f)+\kappa^{-1} \psi_{i-1,0}\left[D_{c}^{i}, f\right]_{c}
$$

and

$$
\psi_{i+j-1,0}\left[D_{c}^{i}, \Delta_{c}^{j}\right]=\left(\left(\kappa^{1-i} \mathrm{id}+\psi_{i, c}\right) D_{c}^{i}\right)\left(\psi_{j, 0} \Delta_{c}^{j}\right)-\left(\left(\kappa^{1-j} \mathrm{id}+\psi_{j, c}\right) \Delta_{c}^{j}\right)\left(\psi_{i, 0} D_{c}^{i}\right) .
$$

When writing (20) for $i=2$, we get

$$
\psi_{2, c} D_{c}^{2}=\left(1-\kappa^{-1}\right) D_{c}^{2}+\kappa^{-1} \beta\left[D_{c}^{2}, \cdot\right]_{c} .
$$

Given that $\psi_{2, c} D_{c}^{2} \in \mathcal{D}_{c}^{1}$, we have

$$
(1-\kappa)\left[D_{c}^{2}, f\right]_{c}(g)=\left[\beta\left[D_{c}^{2}, \cdot\right]_{c}, f\right]_{c}(g) .
$$

Since $\pi_{c},[\cdot, \cdot]$ and $\beta$ are local, the same equation holds locally. If $D_{c}^{2}=D_{c}^{1}+D^{i j} \partial_{i j}$, an easy computation shows that

$$
(1-\kappa)\left[D_{c}^{2}, f\right]_{c}(g)=(1-\kappa)\left(D^{i j}+D^{j i}\right) \partial_{i} f \partial_{j} g,
$$

$\beta\left[D_{c}^{2}, f\right]_{c}=\lambda\left(D^{i j}+D^{j i}\right) \partial_{i j} f+\ldots$, where $\ldots$ are terms of the first order in $f$ and

$$
\left[\beta\left[D_{c}^{2}, \cdot\right]_{c}, f\right]_{c}(g)=2 \lambda\left(D^{i j}+D^{j i}\right) \partial_{i} f \partial_{j} g
$$




\section{J. Grabowski and N. Poncin}

so that

$$
1-\kappa=2 \lambda .
$$

Equation (21), written for $i=1$ and $j=2$, reads

$$
\psi_{2,0}\left(L_{X} \Delta\right)=X\left(\psi_{2,0} \Delta\right)-\Delta(\beta X)-\kappa^{-1} \beta[\Delta, \beta X]_{c},
$$

for all $X \in \operatorname{Vect}(M)$ and all $\Delta \in \mathcal{D}_{c}^{2}$.

In order to show that $\psi_{2,0} \in \mathcal{L}\left(\mathcal{D}_{c}^{2}, \mathcal{A}\right)$ is local, note that it follows for instance from [Pon02] (see $\S 3$ ) that, if $D \in \mathcal{D}_{c}^{2}$ vanishes on an open $U \subset M$ and if $x_{0} \in U$, we have $D=\sum_{k} L_{X_{k}} D_{k}$ $\left(X_{k} \in \operatorname{Vect}(M), D_{k} \in \mathcal{D}_{c}^{2}\right)$, with $\left.X_{k}\right|_{V}=\left.D_{k}\right|_{V}=0$, for some neighborhood $V \subset U$ of $x_{0}$. It then suffices to combine this decomposition of $D$ and Equation $(23)$ to find that $\left(\psi_{2,0} D\right)\left(x_{0}\right)=0$.

Let $U$ be a connected, relatively compact domain of local coordinates of $M$, in which the divergence of a vector field has the form (9). Recall that if $\Delta \in \mathcal{D}_{c, U}^{2}$, its representation is a polynomial $\Delta \in C^{\infty}\left(U, \mathbb{R}^{n} \oplus \bigvee^{2} \mathbb{R}^{n}\right)$. Therefore, $\left.\psi_{2,0}\right|_{U} \in \mathcal{L}\left(C^{\infty}\left(U, \mathbb{R}^{n} \oplus \bigvee^{2} \mathbb{R}^{n}\right), C^{\infty}(U)\right)_{\text {loc }}$, with the representation $\psi(\eta ; \Delta)\left(\eta \in\left(\mathbb{R}^{n}\right)^{*}, \Delta \in \mathbb{R}^{n} \oplus \bigvee^{2} \mathbb{R}^{n}\right)$. As is easily checked, Equation (23) locally reads

$$
\begin{aligned}
(X . \psi)(\eta ; \Delta)-\langle X, \eta\rangle \tau_{\zeta} \psi & (\eta ; \Delta)+\psi\left(\eta+\zeta ; X \tau_{\zeta} \Delta\right) \\
& -\lambda\langle X, \zeta\rangle \Delta(\zeta)-\kappa^{-1} \lambda^{2}\langle X, \zeta\rangle(\Delta(\eta+2 \zeta)-\Delta(\eta+\zeta)-\Delta(\zeta))=0,
\end{aligned}
$$

where $\zeta \in\left(\mathbb{R}^{n}\right)^{*}$ once more represents the derivatives acting on $X$ and where $X . \psi$ is obtained by derivation of the coefficients of $\psi$ in the direction of $X$.

Take in $(24)$ the terms of degree zero in $\zeta:(X . \psi)(\eta ; \Delta)=0$. This means that the coefficients of $\psi$ are constant.

The terms of degree one lead to the equation $\langle X, \eta\rangle\left(\zeta \partial_{\eta}\right) \psi(\eta ; \Delta)-\psi\left(\eta ; X\left(\zeta \partial_{\xi}\right) \Delta\right)=0$, which, if $\rho$ denotes the natural action of $g l(n, \mathbb{R})$, may be written $\rho(X \otimes \zeta)(\psi(\eta ; \Delta))=0$. Note that $\psi(\eta ; \Delta)$ is completely characterized by $\psi\left(\eta ; Y^{k}\right)\left(Y \in \mathbb{R}^{n}, k \in\{1,2\}\right)$. This last expression is a polynomial in $\eta$ and $Y$ (remark that it is homogeneous of degree $k$ in $Y$ ). It follows from the description of invariant polynomials under the action of $\operatorname{gl}(n, \mathbb{R})$ (see [Wey46]), that it is a polynomial in the evaluation $\langle Y, \eta\rangle$. Finally,

$$
\psi\left(\eta ; Y^{k}\right)=c_{k}\langle Y, \eta\rangle^{k}
$$

where $c_{k} \in \mathbb{R}$.

Seeking the terms of degree two in $\zeta$, we find

$$
\begin{aligned}
& \frac{1}{2}\langle X, \eta\rangle\left(\zeta \partial_{\eta}\right)^{2} \psi(\eta ; \Delta)-\left(\zeta \partial_{\eta}\right) \psi\left(\eta ; X\left(\zeta \partial_{\xi}\right) \Delta\right)-\frac{1}{2} \psi\left(\eta ; X\left(\zeta \partial_{\xi}\right)^{2} \Delta\right) \\
& =-\lambda\langle X, \zeta\rangle \Delta^{1}(\zeta)-\kappa^{-1} \lambda^{2}\langle X, \zeta\rangle\left(\left(\zeta \partial_{\eta}\right) \Delta(\eta)-\Delta^{1}(\zeta)\right),
\end{aligned}
$$

where $\Delta^{1}(\zeta)$ denotes the terms of degree one in $\Delta(\zeta)$. Substitute now $Y^{k}(k \in\{1,2\})$ to $\Delta$ and observe that $X\left(\zeta \partial_{\xi}\right) Y^{k}=\langle Y, \zeta\rangle\left(X \partial_{Y}\right) Y^{k}$ and $X\left(\zeta \partial_{\xi}\right)^{2} Y^{k}=k\langle Y, \zeta\rangle^{2}\left(X \partial_{Y}\right) Y^{k-1}$. The left-hand side of (26) then reads

$$
\frac{1}{2}\langle X, \eta\rangle\left(\zeta \partial_{\eta}\right)^{2} \psi\left(\eta ; Y^{k}\right)-\langle Y, \zeta\rangle\left(\zeta \partial_{\eta}\right)\left(X \partial_{Y}\right) \psi\left(\eta ; Y^{k}\right)-\frac{k}{2}\langle Y, \zeta\rangle^{2}\left(X \partial_{Y}\right) \psi\left(\eta ; Y^{k-1}\right) .
$$

When setting $k=1$, then $k=2$, when using (25) (if $k=1$, the last term of the left-hand side vanishes) and noting that the evaluations $\langle X, \eta\rangle,\langle X, \zeta\rangle,\langle Y, \eta\rangle$, and $\langle Y, \zeta\rangle$ can be viewed, if $n>1$ as independent variables, we get from Equation (26)

$$
c_{1}=\lambda, \quad c_{1}+c_{2}=0, \quad c_{2}=\kappa^{-1} \lambda^{2} .
$$

If $n=1$, we only find $c_{1}=\lambda$ and $c_{1}+3 c_{2}=2 \kappa^{-1} \lambda^{2}$, but when selecting in (24) the terms of degree 


\section{Automorphisms of Quantum and Classical Poisson algebras}

three in $\zeta$, we get $c_{1}+2 c_{2}=\lambda+2 \kappa^{-1} \lambda^{2}$, so that (27) still holds. The solutions of the system (22), (27) are $\kappa=1, \lambda=0, c_{1}=c_{2}=0$ and $\kappa=-1, \lambda=1, c_{1}=1, c_{2}=-1$.

Let us first examine the case $\kappa=1$. Equation (21), written - more generally - for $i=1$ and $j \geqslant 2$, reads

$$
\psi_{j, 0}\left(L_{X} \Delta^{j}\right)=L_{X}\left(\psi_{j, 0} \Delta^{j}\right), \quad \text { for all } X \in \operatorname{Vect}(M) \text { and all } \Delta^{j} \in \mathcal{D}^{j},
$$

since $\left.\psi_{j}\right|_{\mathcal{A}}=0$, so that $\psi_{j, 0}$ is an intertwining operator from $\left(\mathcal{D}^{j}, L\right)$ into $\left(\mathcal{D}^{0}, L\right)$. The results of [Pon02] or [BHMP02] show that $\psi_{j, 0}=\left.\lambda_{j} \pi_{0}\right|_{\mathcal{D}^{j}}\left(\lambda_{j} \in \mathbb{R}\right.$ ), for all $j \geqslant 2$ (and all $n \geqslant 1$, indeed a straightforward adaptation of the method of [Pon02] immediately shows that this particular result is also valid in dimension $n=1$ ). It is now easy to verify that $\Phi_{2}$ (see (17)) is id and that $\Phi_{1}=e^{\bar{\omega}}$.

If $\kappa=-1$, we have $\left.\psi_{1,0}\right|_{\mathcal{D}_{c}^{1}}=\left.\mathcal{C}_{0}\right|_{\mathcal{D}_{c}^{1}}$, where $\mathcal{C}$ is the automorphism introduced in Lemma 1.

Inductively, if $\left.\psi_{j-1,0}\right|_{\mathcal{D}_{c}^{j-1}}=\left.\mathcal{C}_{0}\right|_{\mathcal{D}_{c}^{j-1}}(j \geqslant 2)$, the same relation holds for $j$. Indeed, we obtain from (20) and (21),

$$
\psi_{j, 0}\left(L_{X} \Delta\right)=L_{X}\left(\psi_{j, 0} \Delta\right)-\Delta\left(\mathcal{C}_{0} X\right)+\mathcal{C}_{0}\left[\Delta, \mathcal{C}_{0} X\right]_{c},
$$

for all $X \in \operatorname{Vect}(M)$ and all $\Delta \in \mathcal{D}_{c}^{j}$. Straightforward computations, using the properties of $\mathcal{C}$, show that

$$
\mathcal{C}_{0}[\Delta, \cdot]_{c}=\Delta-\mathcal{C}_{c} \Delta=\Delta-\mathcal{C} \Delta+\mathcal{C}_{0} \Delta
$$

on $\mathcal{A}$ as $[\Delta, f]_{c}=[\Delta, f]-\Delta(f)(f \in \mathcal{A})$ and that

$$
\mathcal{C}_{0}\left(L_{X} \Delta\right)=L_{X}\left(\mathcal{C}_{0} \Delta\right)-\left(\mathcal{C}_{c} \Delta\right)\left(\mathcal{C}_{0} X\right) .
$$

It follows from (28), (29) and (30), that

$$
\psi_{j, 0}\left(L_{X} \Delta\right)-L_{X}\left(\psi_{j, 0} \Delta\right)=\mathcal{C}_{0}\left(L_{X} \Delta\right)-L_{X}\left(\mathcal{C}_{0} \Delta\right) .
$$

This last equation is still valid for $\Delta \in \mathcal{D}^{j}$ and signifies that $\psi_{j, 0}-\left.\mathcal{C}_{0}\right|_{\mathcal{D}^{j}}$ is an intertwining operator from $\left(\mathcal{D}^{j}, L\right)$ into $\left(\mathcal{D}^{0}, L\right)$. Thus, applying once more the results of [Pon02] or [BHMP02], we see that

$$
\left.\psi_{j, 0}\right|_{\mathcal{D}_{c}^{j}}=\left.\mathcal{C}_{0}\right|_{\mathcal{D}_{c}^{j}}
$$

It is now again easy to prove (use (18) on $\mathcal{D}_{c}^{j},(31),(20)$ and (29)) that $\Phi_{2}=\mathcal{C}$ and that $\Phi_{1}=\mathcal{C} \circ e^{\bar{\omega}}$. Hence, the only automorphisms of $\mathcal{D}$ that coincide with $\kappa \cdot \operatorname{id}(\kappa \in \mathbb{R}, \kappa \neq 0)$ on functions, are $\Phi_{1}=e^{\bar{\omega}}$ (here $\kappa=1$ ) and $\Phi_{1}=\mathcal{C} \circ e^{\bar{\omega}}$ (here $\kappa=-1$ ), where $\omega$ is a closed 1-form on $M$. Summarizing, we get the following characterization.

Theorem 10. A linear map $\Phi: \mathcal{D}(M) \rightarrow \mathcal{D}(M)$ is an automorphism of the Lie algebra $\mathcal{D}(M)$ of linear differential operators on $C^{\infty}(M)$ if and only if it can be written in the form

$$
\Phi=\phi_{*} \circ \mathcal{C}^{a} \circ e^{\bar{\omega}}
$$

where $\phi$ is a diffeomorphism of $M, a=0,1, \mathcal{C}^{0}=\mathrm{id}, \mathcal{C}^{1}=\mathcal{C}$ and $\omega$ is a closed 1-form on $M$. All the objects $\phi, a, \omega$ are uniquely determined by $\Phi$.

Let us finally note that the above theorem states once more that all automorphisms of $\mathcal{D}(M)$ respect the filtration and thus shows that one-parameter groups of automorphisms of the Lie algebra $\mathcal{D}(M)$ (for any reasonable topology on $\mathcal{D}(M)$ ) cannot have as generators the inner derivations $\operatorname{ad}_{D}$ for $D$ not being of first order. An analogous fact holds for the Lie algebra $S(M)$. Thus we have the following. 


\section{J. Grabowski and N. Poncin}

Corollary 4. The Lie algebras $\mathcal{D}(M)$ and $S(M)$ of linear differential operators on $C^{\infty}(M)$, and of the principal symbols of these operators, are not integrable, i.e. there are no (infinite-dimensional) Lie groups for which they are the Lie algebras.

\section{REFERENCES}

Abe82 K. Abe, Pursell-Shanks type theorem for orbit spaces and G-manifolds, Publ. Res. Inst. Math. Sci. 18 (1982), 265-282.

Ame75 I. Amemiya, Lie algebra of vector fields and complex structure, J. Math. Soc. Japan 27 (1975), 545-549.

AG90 C. J. Atkin and J. Grabowski, Homomorphisms of the Lie algebras associated with a symplectic manifold, Compositio Math. 76 (1990), 315-348.

BHMP02 F. Boniver, S. Hansoul, P. Mathonet and N. Poncin, Equivariant symbol calculus for differential operators acting on forms, Lett. Math. Phys. 62 (2002), 219-232.

DWL83 M. De Wilde and P. Lecomte, Cohomology of the Lie algebra of smooth vector fields of a manifold, associated to the Lie derivative of smooth forms, J. Math. Pures Appl. 62 (1983), 197-214.

Gra78 J. Grabowski, Isomorphisms and ideals of the Lie algebras of vector fields, Invent. Math. 50 (1978), 13-33.

Gra93 J. Grabowski, Lie algebras of vector fields and generalized foliations, Publ. Mat. 37 (1993), 359-367.

Gra00 J. Grabowski, Isomorphisms of Poisson and Jacobi brackets, in Poisson Geometry, eds J. Grabowski and P. Urbański, Banach Center Publications, vol. 51 (Banach Center, Warszawa, 2000), 79-85.

HM93 H. Hauser and G. Müller, Affine varieties and Lie algebras of vector fields, Manusc. Math. 80 (1993), 309-337.

Lec02 P. Lecomte, Sur les champs de densités sur une variété, seminar notes (2002), Université de Liège (electronic version at http://www.ulg.ac.be/geothalg/QPE/index.html).

LMT96 P. Lecomte, P. Mathonet and E. Tousset, Comparison of some modules of the Lie algebra of vector fields, Indag. Math. 7 (1996), 461-471.

LO99 P. Lecomte and V. Ovsienko, Projectively equivariant symbol calculus, Lett. Math. Phys. 49 (1999), 173-196.

Omo76 H. Omori, Infinite dimensional Lie transformation groups, Lecture Notes in Mathematics, vol. 427 (Springer, Berlin, 1976).

Pee59 J. Peetre, Une caractérisation abstraite des opérateurs différentiels, Math. Scand. 7 (1959), 211-218; 8 (1960), 116-120.

Pon01 N. Poncin, On the cohomology of the Nijenhuis-Richardson graded Lie algebra of the space of functions of a manifold, J. Algebra 243 (2001), 16-40.

Pon02 N. Poncin, Equivariant operators between some modules of the Lie algebra of vector fields, Centre Universitaire de Luxembourg Preprint (2002)

(electronic version at http://arXiv.org/abs/math.RT/0205297).

PS54 M. E. Shanks and L. E. Pursell, The Lie algebra of a smooth manifold, Proc. Amer. Math. Soc. 5 (1954), 468-472.

Skr87 S. M. Skryabin, The regular Lie rings of derivations of commutative rings, Preprint WINITI 4403-W87 (1987). A summary can be found in S. M. Skryabin, Vestnik Moscov. Univ. Ser. I. Mat. Mekh. 3 (1988), 59-62 [Moscow Univ. Math. Bull. 43 (1988), 56-58].

Vin72 A. M. Vinogradov, The logic algebra for the theory of linear differential operators, Sov. Mat. Dokl. 13 (1972), 1058-1062.

Wey46 H. Weyl, The classical groups, their invariants and representations (Princeton University Press, Princeton, NJ, 1997). 


\section{Automorphisms of quantum and Classical Poisson algebras}

J. Grabowski jagrab@impan.gov.pl

Polish Academy of Sciences, Institute of Mathematics, ul. 'Sniadeckich 8, PO Box 137, 00-950 Warsaw, Poland

N. Poncin poncin@cu.lu

Université de Luxembourg, Département de Mathématiques, avenue de la Faïencerie, 162 A, L-1511 Luxembourg, Luxembourg 